\title{
Prevalence and Self-recognition of Chronic Constipation: Results of an Internet Survey
}

\begin{abstract}
Akio Tamura, Toshihiko Tomita, Tadayuki Oshima, Fumihiko Toyoshima, Takahisa Yamasaki, Takuya Okugawa, Takashi Kondo, Tomoaki Kono, Katsuyuki Tozawa, Hisatomo Ikehara, Yoshio Ohda, Hirokazu Fukui, Jiro Watari, and Hiroto Miwa*
\end{abstract}

Division of Gastroenterology, Department of Internal Medicine, Hyogo College of Medicine, Hyogo, Japan

\section{Background/Aims}

Although chronic constipation is a common symptom, to date no international consensus has been reached regarding its definition. The aims of this study were (1) to investigate defecation habits and (2) to examine the prevalence of constipation using the Japanese Society of Internal Medicine (JSIM) and the Rome III criteria using an online survey.

\section{Methods}

An online questionnaire composed of items on the frequency, interval, form of defecation, the management, and self-recognition of constipation (reference standard of constipation) was created. A total of 5155 valid responses were received. In addition, constipation symptoms were evaluated through a survey using the JSIM and the Rome III criteria.

\section{Results}

In the internet survey, $28.4 \%$ of the respondents considered themselves to be constipated. Stratified by sex, significantly more females $(37.5 \%)$ than males $(19.1 \%)$ considered themselves to be constipated $(P<0.001)$. The prevalence of constipation among the respondents was $28.0 \%$ using the Rome III, but only $10.1 \%$ using the JSIM. The diagnostic accuracy was $73.2 \%$ for the Rome III and $78.1 \%$ for the JSIM, while the diagnostic specificity was $81.1 \%$ for the Rome III and $97.5 \%$ for the JSIM. However, the diagnostic sensitivities for both measures were low, at $52.2 \%$ and $29.2 \%$ for the Rome III and the JSIM, respectively.

\section{Conclusions}

The online survey developed for this study was able to provide clarification regarding defecation patterns. The results also suggest a discrepancy between the self-recognized prevalence of constipation in Japan and prevalence of constipation based on the JSIM criteria.

(J Neurogastroenterol Motil 2016;22:677-685)

Key Words

Constipation; Prevalence; Recognition

Received: November 28, 2015 Revised: May 9, 2016 Accepted: June 2, 2016

(a) This is an Open Access article distributed under the terms of the Creative Commons Attribution Non-Commercial License (http://creativecommons. org/licenses/by-nc/4.0) which permits unrestricted non-commercial use, distribution, and reproduction in any medium, provided the original work is properly cited.

${ }^{*}$ Correspondence: Hiroto Miwa, MD, PhD

Division of Gastroenterology, Department of Internal Medicine, Hyogo College of Medicine, 1-1, Mukogawa-cho, Nishinomiya, Hyogo 663-8501, Japan Tel: +81-798-45-6665, Fax: +81-798-45-6661, E-mail: miwahgi@hyo-med.ac.jp 


\section{Introduction}

Chronic constipation is a condition frequently encountered in the clinical setting. ${ }^{1-4}$ However, the definition and understanding of constipation vary widely among individuals, and in some cases, by region. ${ }^{5-8}$ The prevalence of constipation based on the Rome III criteria has been reported at varying levels around the world, from $8.2 \%$ to $32.9 \% .^{9-17}$ In Asian countries such as China, Korea, Hong Kong, and India, the prevalence of constipation reportedly ranges from $8.2 \%$ to $16.8 \% .^{9,10,12,13}$ However, little epidemiological information regarding defecation habits and the prevalence of constipation in Japan is currently available.

Epidemiological surveys conducted by conventional techniques are becoming more difficult to carry out due to enhanced protection of personal privacy. However, an epidemiological internet survey can provide a great deal of data without requiring individuals to identify themselves. In fact, according to the recent Communications Usage Trend Survey by the Japanese Ministry of Internal Affairs and Communications (MIC), over 100 million Japanese citizens now have access to and frequently use the internet. Furthermore, because the sample population chosen for participation in an internet survey can be selected to be representative of the Japanese population as a whole, the results are expected to be highly reliable predictors of the actual situation in Japan.

Therefore, we conducted an internet survey (1) to investigate the defecation habits and attitudes toward constipation in Japan, and (2) to examine the prevalence of constipation based on the Japanese Society of Internal Medicine (JSIM) and the Rome III criteria.

\section{Materials and Methods}

\section{Internet Questionnaire Survey}

With the cooperation of an internet research company (Macromill, Inc, Tokyo, Japan), we conducted an internet questionnaire survey in August 2014 targeting males and females of the general
Japanese population aged 20 to 79 years. We hoped to collect data from approximately 5000 respondents; therefore, we asked a total of 15000 individuals to participate. Respondents who failed to completely answer the questions were excluded from the analysis. A total of 5155 subjects (2542 males and 2613 females) who completed the questionnaire were finally enrolled. Male and female subjects in each age group (20 to < 30 years, 20's; 30 to < 40 years, 30's; 40 to $<50$ years, 40's; 50 to $<60$ years, 50's; 60 to $<70$ years, 60 's; and 70 to $<80$ years, 70's) were selected from among the registered panelists based on Japanese demographics (Table 1). The respondents in this study were assigned to groups on the internet in reference to the population ratio in Japan by generation and sex according to the national census conducted by the MIC Statistics Bureau (2010).

\section{Questionnaire Items}

We developed an original questionnaire to diagnose constipation in accordance with the Rome III (Japanese version) and the JSIM criteria. ${ }^{18}$ The questionnaire was composed of 13 items regarding defecation habits and actual status. The questionnaire items are shown in the Appendix. The participants were asked about their recognition of constipation, frequency of defecation, stool appearance, typical defecation interval, and if they had symptoms that met the diagnostic criteria for irritable bowel syndrome (IBS). ${ }^{19-21}$ Selfrecognition of constipation was set as the reference standard of constipation in this study. We also asked respondents who recognized themselves as being constipated about how they managed their constipation.

\section{Statistical Methods}

All results are expressed as mean \pm standard deviation. The data were assessed by the Mann-Whitney U-test and Fisher's exact test. Statistical significance was defined as $P<0.05$.

Table 1. Characteristics of the Subjects

\begin{tabular}{lccccccc}
\hline \multicolumn{1}{c}{ Age group } & $20-29 \mathrm{yr}$ & $30-39 \mathrm{yr}$ & $40-49 \mathrm{yr}$ & $50-59 \mathrm{yr}$ & $60-69 \mathrm{yr}$ & $70-79 \mathrm{yr}$ & Total \\
\hline Number (M/F) & $367 / 355$ & $490 / 476$ & $453 / 445$ & $437 / 442$ & $479 / 509$ & $316 / 386$ & $2542 / 2613$ \\
Age (M/F), mean $\pm \mathrm{SD}(\mathrm{yr})$ & $24.9 \pm 2.8 /$ & $35.1 \pm 2.9 /$ & $44.6 \pm 2.8 /$ & $54.0 \pm 2.8 /$ & $64.1 \pm 2.6 /$ & $73.2 \pm 2.7 /$ & $48.8 \pm 15.9 /$ \\
& $25.3 \pm 2.8$ & $34.8 \pm 2.9$ & $44.3 \pm 2.8$ & $53.9 \pm 2.8$ & $63.8 \pm 2.5$ & $72.9 \pm 2.6$ & $49.6 \pm 16.0$ \\
\hline
\end{tabular}




\section{Results}

\section{Actual Status of Defecation-related Issues in Japan (Frequency of Defecation, Stool Appearance, Sense of Incomplete Defecation, Sense of Difficult Defecation)}

The mean overall frequency of defecations per week was $6.9 \pm$ 4.2. A total of $8.4 \%$ of all respondents reported that they defecated less than twice a week, and $25.0 \%$ reported that they defecated more than 8 times a week (Fig. 1A). Stratified by sex, the mean was significantly lower in females $(5.9 \pm 3.4)$ than in males $(7.9 \pm 4.7)$ $(P<0.001)$ (Fig. 1B). Stratification by age produced no significant differences between age groups in men. Females in their 20's had the lowest mean frequency of defecations per week $(5.1 \pm 2.8)$, with this frequency increasing significantly with increasing age.

Stool appearance was defined using the Bristol scale, with values of 1 or 2 considered to be "hard" stool, 3, 4, and 5 considered to be "normal", and 6 and 7 considered to be "soft" ${ }^{22-24}$ Among all respondents, the prevalence of "hard", "normal", and "soft" stool was $19.3 \%$ (997/5155), 67.6\% (3483/5155), and 13.1\% (674/5155), respectively. Significantly more females $(652 / 2613 ; 25.0 \%)$ than males $(345 / 2542 ; 13.6 \%)$ reported having hard stool. The frequency of hard stool in females increased significantly with younger age. Among respondents with "hard stool", 51.1\% (509/997) were diagnosed as having functional constipation (FC) and 16.0\% (160/997) as IBS with constipation (IBS-C).

A sense of incomplete defecation was reported by $36.5 \%$ (1879/5155) of the respondents, and a sense of difficult defecation (straining at stool, sensation of blockage, and use of measures such as pressing the abdomen during defecation) was reported by $54.8 \%$

\section{Perceptions of Constipation}

Constipation was only considered to be a medical condition by approximately half (48.8\%) of the respondents. When asked what constitutes constipation, the largest number of respondents (3644/5155; 70.7\%) replied, "Unable to defecate without medication." The next most common response $(3549 / 5155 ; 68.8 \%)$ was, "Few bowel movements", and the third and fourth most common responses were "Difficulty in defecating" (3533/5155; 68.5\%) and "Hard stool" (3007/5155; 58.3\%), respectively. In the respondents with self-recognized constipation, "Few bowel movements (1215/1464; 83.0\%)", "Difficulty in defecating (1124/1464; $76.8 \%)$ ", "Hard stool (1046/1464; 71.4\%)", "Unable to defecate without medication (979/1464; 66.9\%)" were considered important. In contrast, in the respondents who did not consider themselves as being constipated, "Few bowel movements (2334/3691; 63.2\%)", "Difficulty in defecating (2409/3691; 65.3\%)", "Hard stool (1961/3691; 53.1\%)", and "Unable to defecate without medication $(2665 / 3691 ; 72.2 \%)$ ” were also considered important.

\section{Percentage of Respondents Who Considered Themselves to Have Constipation}

A total of 28.4\% (1464/5155) of the respondents thought that they had constipation (Fig. 2A). When stratified by sex, significantly more females $(979 / 2613 ; 37.5 \%)$ than males $(485 / 2542 ; 19.1 \%)$ thought that they had constipation $(P<0.001)$ (Fig. 2B). When stratified by sex and age group, no significant differences between age groups were noted in men. The percentage of women who considered themselves to have constipation increased with younger age $(P<0.001)$. In the respondents with "hard stool", 68.9\% (687/997) thought that they had constipation.
A

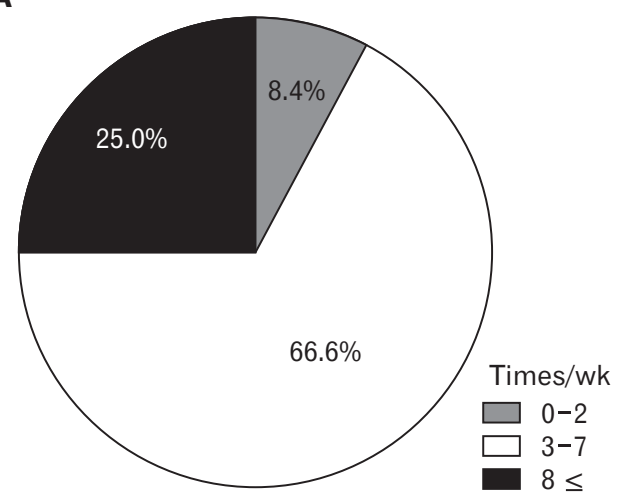

B

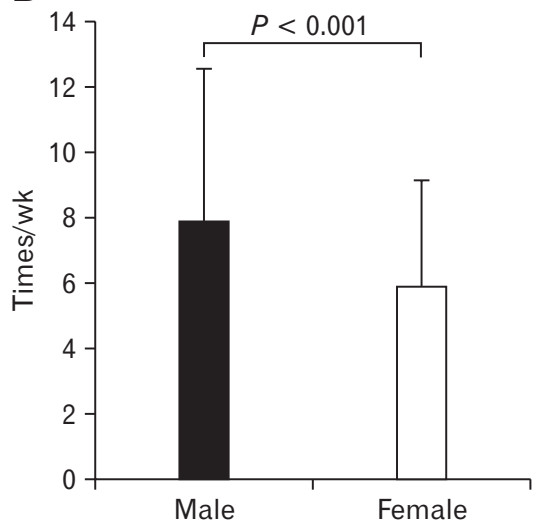

Figure 1. Actual status of defecationrelated issues. (A) Frequency of defecations per week among the respondents $(\mathrm{n}=5155)$. (B) Number of defecations per week in each sex. 
A

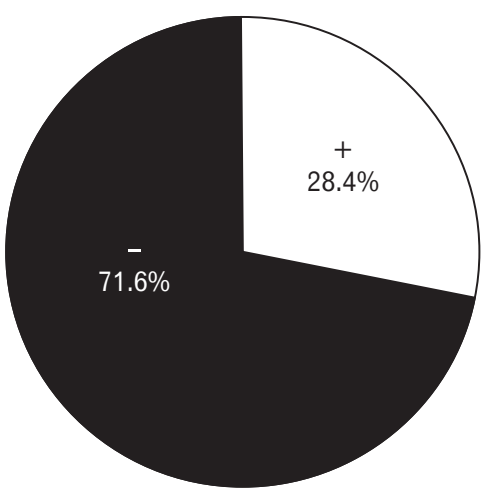

Self-recognized constipation

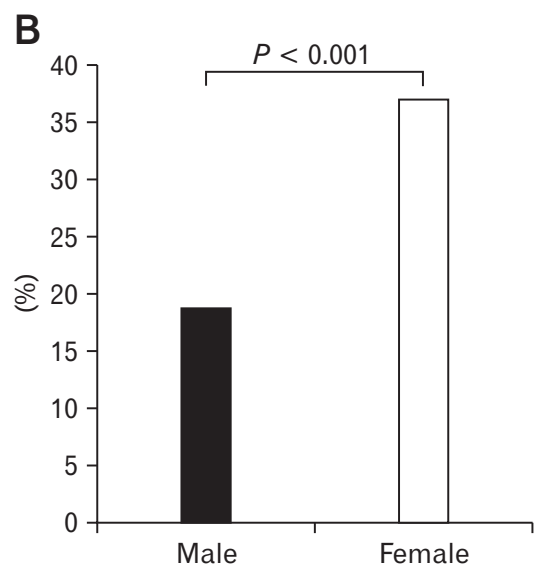

Figure 2. Percentage of respondents who considered themselves as being constipated $(\mathrm{n}=5155)$. (A) Prevalence of selfrecognition of constipation. (B) Ratio of self-recognition of constipation in each sex.
A

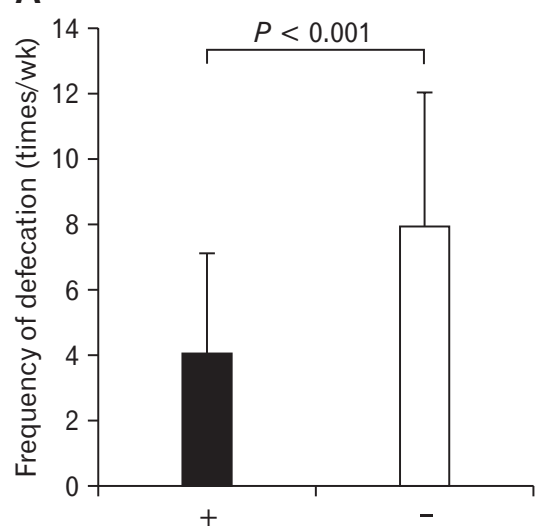

B

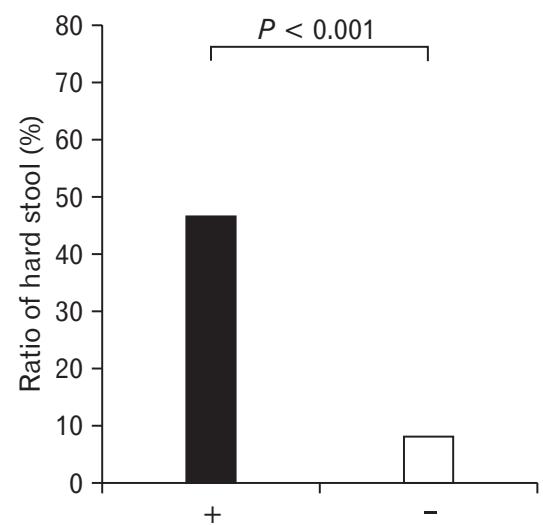

C

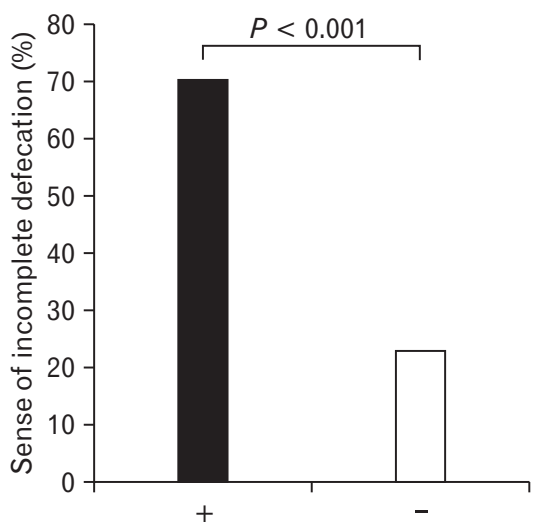

Figure 3. Differences in stool characteristics with and without self-recognized constipation. (A) Frequency of defecations per week in respondents with (+) and without self-recognized constipation (-). (B) Ratio of hard stool (Bristol stool forms 1 and 2). (C) Sense of incomplete defecation.

\section{Differences in Stool Characteristics among Respondents with and Without Self-recognized Constipation}

The respondents who considered themselves as being constipated reported a mean of 4.2 bowel movements per week, compared with 8.0 among those who did not $(P<0.001)$ (Fig. 3A). The frequency of hard stool was $46.9 \%$ and $8.4 \%$, respectively $(P<0.001)$ (Fig. 3B). The ratio of respondents who reported experiencing a sense of incomplete defecation was $70.3 \%$ and $23.0 \%$, respectively $(P$ $<0.001$ ) (Fig. 3C).

\section{Management of Constipation}

Increased water intake was the most commonly held idea as a countermeasure against constipation $(52.2 \%, 2693 / 5155$ respondents). The next most common idea was to get more sleep (39.7\%), followed by changes in diet (38.6\%), use of a bidet-toilet (37.1\%), reduction of stress $(31.0 \%)$, and exercise $(25.3 \%)$. Over-thecounter oral medicines were used by $7.9 \%$. Only a few respondents $(4.7 \%)$ had seen a doctor for their condition. Most of the males and females who saw a doctor for constipation were elderly (Fig. 4).

\section{Prevalence of Constipation in the Japanese} Population According to the Rome III and Japanese Society of Internal Medicine Criteria

A total of $28.0 \%$ (1445/5155) of the respondents were classified as having FC based on the Rome III criteria. In contrast, only $10.1 \%(520 / 5155)$ met the criteria for constipation as defined by the JSIM.

Of the respondents who considered themselves as being constipated, 52.2\% (764/1464) and 29.2\% (428/1464) met the Rome III and JSIM diagnostic criteria, respectively. The diagnostic ratio 
according to the Rome III and JSIM criteria is shown in Table 2. The diagnostic accuracy was $73.2 \%$ and $78.1 \%$ for the Rome III and JSIM criteria, respectively, while the diagnostic specificity was $81.5 \%$ and $97.5 \%$ for the Rome III and JSIM criteria, respectively. However, the diagnostic sensitivity was low for both measures, at $52.2 \%$ and $29.2 \%$, respectively.

\section{The Subtype of the Subjects with Self-recognized Constipation}

Approximately half $(52.2 \%, 764 / 1464)$ of the respondents with self-recognized constipation were diagnosed as having FC, one-quarter $(23.8 \%$; $9.5 \%$ IBS-C, $6.7 \%$ mixed IBS, $4.3 \%$ IBS with diarrhea, and $3.3 \%$ unsubtyped IBS) were diagnosed with IBS, and the remainder (24.0\%) were diagnosed with another condition (Fig. 5). All participants (100\%, 1455/1455) with IBS-C responded as having Bristol scale stool forms 1 and 2; however, only one third (35.2\%) of those with FC responded as having Bristol scale stool forms 1 and 2 .

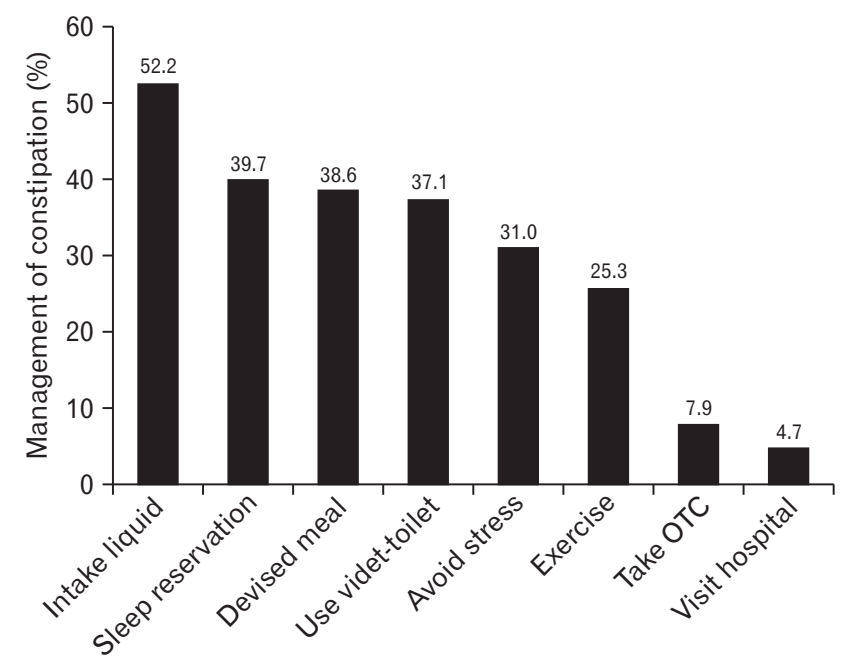

Figure 4. Constipation management $(\mathrm{n}=5155)$. OTC, over the counter.

\section{Discussion}

Constipation is characterized by infrequent bowel movements or difficulty in defecation. Constipation is generally diagnosed from symptoms such as a reduced number of weekly bowel movements, hard stool, a sense of incomplete defecation or a sense of difficult defecation. ${ }^{25-28}$ However, constipation is recognized differently among individuals, regions, and cultures. ${ }^{5-8}$ Therefore, in order to investigate the actual status of constipation in Japan, it is first necessary to clearly understand how Japanese people conceptualize constipation and their defecation habits. For this purpose, we conducted an internet survey to investigate how constipation was recognized among Japanese people. Our results clearly showed that only about half of the respondents considered constipation to be a medical condition. This situation was evidenced by the fact that the most common countermeasures among respondents who developed constipation were lifestyle adjustments such as drinking more water, getting more sleep, and changing their diet. Only $4.7 \%$ of the respondents actually visited their doctor for constipation.

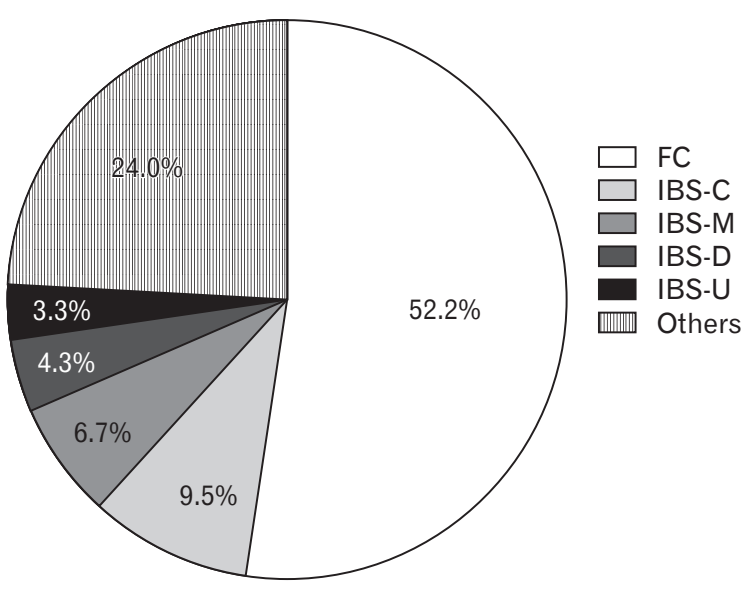

Figure 5. The subtypes of respondents with self-recognized constipation. FC, functional constipation; IBS, irritable bowel syndrome; IBSU, unsubtyped IBS; IBS-D, IBS with diarrhea; IBS-C, IBS with constipation; IBS-M, mixed IBS.

Table 2. Diagnostic Accuracy Between the Definitions by the Japanese Society of Internal Medicine and the Rome III Criteria

\begin{tabular}{lccccc}
\hline & Sensitivity & Specificity & Accuracy & PPV & NPV \\
\hline JSIM & 0.292 & 0.975 & 0.781 & 0.823 & 0.776 \\
Rome III & 0.522 & 0.815 & 0.732 & 0.529 & 0.811 \\
\hline
\end{tabular}

PPV, positive predictive value; NPV, negative predictive value; JSIM, the Japanese Society of Internal Medicine. 
When we investigated defecation habits, we found that respondents reported a mean of 6.9 bowel movements per week. The most common range (66.5\% of respondents) was 3-7 bowel movements per week, while a relatively small number had 0-2 movements per week. When asked about stool appearance, most of the responders reported normal stool, and $19.3 \%$ reported lumpy or hard stool. Moreover, a sense of incomplete defecation was reported by $36.5 \%$ of the respondents, and a sense of difficult defecation (straining, sensation of anorectal obstruction/blockage, or manual maneuvers) was reported by $54.8 \%$. These are valuable data since no detailed information concerning actual defecation habits in Japan has been available to date.

So what constitutes constipation in the opinion of the Japanese people? To answer this question, we compared the characteristics of defecation in respondents who considered themselves to be constipated with those who did not. Among the respondents who had no more than two bowel movements per week, approximately $80 \%$ considered themselves to be constipated. With regard to defecation characteristics, we found that $68.9 \%$ of the respondents with hard stool and $70.3 \%$ of those who had a sense of incomplete defecation thought that they were constipated. These findings suggest that the number of bowel movements, the hardness of stool, and a sense of incomplete defecation are all associated with constipation. In both respondents with and without self-recognized constipation, few bowel movements, difficulty in defecating, hard stool, and unable to defecate without medication were most important senses of constipation. These results indicate that individuals only consider themselves to be constipated when they have a combination of conditions, as opposed to any one specific characteristic.

This present study allows us to evaluate whether constipation is appropriately defined in Japan. A wide variety of definitions of constipation are used around the world, several of which are used in Japan. ${ }^{29-31}$ For example, the JSIM defines constipation as " 3 or more days without a bowel movement, or a sense of incomplete defecation after daily bowel movements". On the international level, the Rome III criteria are widely used as a definition of functional constipation. ${ }^{32}$ These criteria require that symptoms have occurred over the previous 6 months, that for the previous 3 months the subject has experienced less than three bowel movements per week and/or symptoms of straining during defecation, hard stool, sense of incomplete defecation, or sense of difficult defecation at least once every four bowel movements, and that the subject does not meet the diagnostic criteria for IBS. However, it is difficult to distinguish between the FC as defined by the Rome III criteria and IBS-C. In the present study, among approximately half of the respondents with self-recognized constipation who were diagnosed as having FC, $23.8 \%$ were diagnosed as having IBS. These results indicate the possibility of overlap, and some reports have even suggested that FC and IBS-C are the same condition. ${ }^{33-36}$

To date, there is no standard definition of constipation. In this survey, using the personal judgment of the respondents as the "gold standard", we assessed the diagnostic accuracy of the definitions of constipation according the Rome III and the JSIM criteria. As a result, no major differences were observed between these definitions in diagnostic accuracy ( $73.2 \%$ vs $78.1 \%$, respectively). However, the JSIM definition provided a diagnostic sensitivity of $29.2 \%$, which was considerably lower than the $52.2 \%$ for the Rome III. The relatively low sensitivity of the JSIM may be attributed to the fact that the definition of constipation included only the prevalence of bowel movements and a sense of incomplete defecation, while omitting stool hardness and a sense of difficult defecation such as straining, sensation of anorectal obstruction/blockage, and manual maneuvers. The relatively low sensitivity (52.2\%) of the Rome III criteria may be attributed to the fact that, although this classification uses a multi-sided definition of constipation (number of bowel movements, hardness of stool, sense of incomplete defecation, sense of difficult defecation, etc), some patients with constipation may instead be diagnosed with IBS. In fact, $23.8 \%$ of the respondents who felt constipated were diagnosed as having IBS. Specificity was $97.5 \%$ for the JSIM criteria, which was markedly higher than the $81.5 \%$ for the Rome III criteria, although both values were high. This may be because the JSIM criteria have few diagnostic items, which resulted in diagnosing a narrower range of patients. If the Rome III definition was applied to all respondents, $28.0 \%$ of the Japanese population would be considered to have constipation, whereas if the JSIM definition was applied, that number would be $10.1 \%$.

In the present study, the prevalence of respondents who reported being constipated was close to the percentage suggested by the Rome III definition. In Asian countries, the prevalence of constipation is reportedly about $8 \%$ to $17 \% .^{9,10,12,13}$ These differences may have been because the study methodologies and backgrounds of the enrolled subjects were different. Moreover, it should be noted that constipation was diagnosed by the Rome III definition in only half of the respondents who self-identified as having constipation. As shown in Figure 5, under the Rome III criteria, 23.8\% of the respondents who reported being constipated would be classified as having IBS, and the remaining respondents were diagnosed with other conditions. In other words, these people were not aware that they had IBS. Further study of IBS in Japan is warranted. 
We conducted this research on the internet. As previously described, this method involves a number of issues, including the completion rate. The completion rate of enrolled subjects in this study was about $30 \%$; although this was not very high, it is in line with the average completion rate of respondents in internet surveys. Moreover, this methodology is limited to internet users, and therefore may only collect data from a specific stratum of subjects, does not guarantee that the survey was actually completed by the subject, and cannot exclude the possibility that subjects might provide casual answers. However, the importance of protecting personal information has become more widely recognized in recent years, which has made it increasingly difficult to conduct large-scale epidemiological studies. In addition, based on communication dynamics research by the MIC 2014, internet users represent about $80 \%$ of the Japanese population. In addition, the internet offers the advantage of obtaining data from respondents aged 20 to 80 years stratified by various characteristics such as sex, age, and regional distribution. Therefore, the results can still be considered credible.

The limitation of this study was the lack of validation of the questionnaire. In the present study, we developed an original questionnaire in accordance with the Rome III and the JSIM criteria. In addition, as the present study was a questionnaire survey for the general population, we could not rule out the inclusion of a variety of conditions in addition to FC (chronic idiopathic constipation), including organic constipation, drug-induced constipation, and constipation secondary to underlying disease. Moreover, we also cannot be sure whether the self-recognition of constipation was referring to chronic constipation or recent constipation.

However, because the survey involved very large sample size of more than 5000 respondents based on Japanese demographics, the findings may still provide useful information regarding the awareness and actual situation of constipation in Japan.

In conclusion, our results clarified (1) defecation habits in Japan, and (2) identified a discrepancy between the prevalence of selfrecognized constipation and that based on the JSIM criteria.

\section{Financial support: None.}

\section{Conflicts of interest: None.}

Author contributions: Akio Tamura and Toshihiko Tomita contributed to the concept and design of the study; Akio Tamura, Toshihiko Tomita, Fumihiko Toyoshima, Takahisa Yamasaki, Takuya Okugawa, Takashi Kondo, Tomoaki Kono, Katsuyuki Tozawa, Hisatomo Ikehara, and Yoshio Ohda were involved in data acquisition and analysis; Tadayuki Oshima provided statistifical design; Jiro Watari and Hiroto Miwa coordinated acquisition of data and trial supervision; and all authors contributed to data interpretation, critically reviewed the manuscript for important intellectual contents, and approved final version of the manuscript.

\section{References}

1. Koloski NA, Talley NJ, Boyce PM. Epidemiology and health care seeking in the functional GI disorders: a population-based study. Am J Gastroenterol 2002;97:2290-2299.

2. Drossman DA, Li Z, Andruzzi E, et al. U.S. householder survey of functional gastrointestinal disorders. Prevalence, sociodemography, and health impact. Dig Dis Sci 1993;38:1569-1580.

3. Stewart WF, Liberman JN, Sandler RS, et al. Epidemiology of constipation (EPOC) study in the United States: relation of clinical subtypes to sociodemographic features. Am J Gastroenterol 1999;94:3530-3540.

4. Johanson JF, Kralstein J. Chronic constipation: a survey of the patient perspective. Aliment Pharmacol Ther 2007;25:599-608

5. Cummings JH, Bingham SA, Heaton KW, Eastwood MA. Fecal weight, colon cancer risk, and dietary intake of nonstarch polysaccharides (dietary fiber). Gastroenterology 1992;103:1783-1789.

6. Sandler RS, Drossman DA. Bowel habits in young adults not seeking health care. Dig Dis Sci 1987;32:841-845.

7. Everhart JE, Go VL, Johannes RS, Fitzsimmons SC, Roth HP, White LR. A longitudinal survey of self-reported bowel habits in the United States. Dig Dis Sci 1989;34:1153-1162.

8. Davies GJ, Crowder M, Reid B, Dickerson JW. Bowel function measurements of individuals with different eating patterns. Gut 1986;27:164169

9. Chu H, Zhong L, Li H, Zhang X, Zhang J, Hou X. Epidemiology characteristics of constipation for general population, pediatric population, and elderly population in china. Gastroenterol Res Pract 2014;2014:532734.

10. Jun DW, Park HY, Lee OY, et al. A population-based study on bowel habits in a Korean community: prevalence of functional constipation and self-reported constipation. Dig Dis Sci 2006;51:1471-1477.

11. Johanson JF, Kralstein J. Chronic constipation: a survey of the patient perspective. Aliment Pharmacol Ther 2007;25:599-608.

12. Rajput M, Saini SK. Prevalence of constipation among the general population: a community-based survey from India. Gastroenterol Nurs 2014;37:425-429.

13. Cheng C, Chan AO, Hui WM, Lam SK. Coping strategies, illness perception, anxiety and depression of patients with idiopathic constipation: a population-based study. Aliment Pharmacol Ther 2003;18:319-326.

14. Higgins PD, Johanson JF. Epidemiology of constipation in North America: a systematic review. Am J Gastroenterol 2004;99:750-759.

15. Pare P, Ferrazzi S, Thompson WG, Irvine EJ, Rance L. An epidemiological survey of constipation in Canada: definitions, rates, demographics, and predictors of health care seeking. Am J Gastroenterol 2001;96:31303137. 
16. Adibi P, Behzad E, Pirzadeh S, Mohseni M. Bowel habit reference values and abnormalities in young Iranian healthy adults. Dig Dis Sci 2007;52:1810-1813.

17. Peppas G, Alexiou VG, Mourtzoukou E, Falagas ME. Epidemiology of constipation in Europe and Oceania: a systematic review. BMC Gastroenterol 2008;8:5.

18. Kanazawa M, Nakajima S, Oshima T, et al. Validity and reliability of the Japanese version of the Rome III diagnostic questionnaire for irritable bowel syndrome and functional dyspepsia. J Neurpgastroenterol Motil 2015;21:537-544.

19. Fukudo S, Kaneko H, Akiho H, et al. Evidence-based clinical practice guidelines for irritable bowel syndrome. J Gastroenterol 2015;50:11-30.

20. Longstreth GF, Drossman DA. New developments in the diagnosis and treatment of irritable bowel syndrome. Curr Gastroenterol Rep 2002;4:427-434

21. Rome Foundation. Guidelines--Rome III diagnostic criteria for functional. gastrointestinal disorders. J Gastrointestin Liver Dis 2006;15:307312.

22. Longstreth GF, Thompson WG, Chey WD, Houghton LA, Mearin F, Spiller RC. Functional bowel disorders. Gastroenterology 2006;130:1480-1491.

23. Barnett JL, Hasler WL, Camilleri M. American Gastroenterological Association medical position statement on anorectal testing techniques. American Gastroenterological Association. Gastroenterology 1999;116:732-760.

24. McCrea GL, Miaskowski C, Stotts NA, Macera L, Varma MG. Pathophysiology of constipation in the older adult. World J Gastroenterol 2008; 7;14:2631-2638.

25. Lembo A, Camilleri M. Chronic constipation. N Engl J Med 2003;349: 1360-1368.

26. Locke GR 3rd, Pemberton JH, Phillips SF. AGA technical review on constipation. American Gastroenterological Association. Gastroenterology 2000;119:1766-1778.
27. Koch A, Voderholzer WA, Klauser AG, Müller-Lissner S. Symptoms in chronic constipation. Dis Colon Rectum 1997;40:902-906.

28. Herz MJ, Kahan E, Zalevski S, Aframian R, Kuznitz D, Reichman S. Constipation: a different entity for patients and doctors. Fam Pract 1996;13:156-159.

29. Tian H, Ding C, Gong J, et al. An appraisal of clinical practice guidelines for constipation: a right attitude towards to guidelines. BMC Gastroenterol 2016;16:52.

30. CADTH Rapid Response Reports. Dioctyl sulfosuccinate or docusate (calcium or sodium) for the prevention or management of constipation: a review of the clinical effectiveness. Ottawa: Canadian Agency for Drugs and Technologies in Health 2014

31. Lindberg G, Hamid SS, Malfertheiner P, et al. World Gastroenterology Organisation global guideline: constipation--a global perspective. J Clin Gastroenterol 2011;45:483-487.

32. Ghoshal UC, Gwee KA, Chen M, et al. Development, translation and validation of enhanced asian Rome III questionnaires for diagnosis of functional bowel diseases in major asian languages: a rome foundationasian neurogastroenterology and motility association working team report. J Neurogastroenterol Motil 2015;21:83-92.

33. Brandt LJ, Prather CM, Quigley EM, Schiller LR, Schoenfeld P, Talley NJ. Systematic review on the management of chronic constipation in North America. Am J Gastroenterol 2005;100(suppl 1):S5-S21.

34. Talley NJ. Differentiating functional constipation from constipationpredominant irritable bowel syndrome: management implications. Rev Gastroenterol Disord 2005;5:1-9.

35. Koloski NA, Jones M, Young M, Talley NJ. Differentiation of functional constipation and constipation predominant irritable bowel syndrome based on Rome III criteria: a population-based study. Aliment Pharmacol Ther 2015;41:856-866.

36. Park JM, Choi MG, Cho YK, et al. Functional gastrointestinal disorders diagnosed by Rome III questionnaire in Korea. J Neurogastroenterol Motil 2011;17:279-286. 